\title{
The Study of Receiver Baseband Algorithm in Space Borne ADS-B (1090ES)
}

\author{
Huan $\mathrm{Li}^{1, \mathrm{a}}$, Dengmao Zhang ${ }^{2, \mathrm{~b}}$, Weizhen $\mathrm{Chen}^{3, \mathrm{c}}$, Yubai $\mathrm{Li}^{4, \mathrm{~d}}$ \\ ${ }^{1}$ The Joint Research Center for Satellite Navigation Sub-centers of The Ministry of Education, \\ University of Electronic Science and Technology of China, Yubai Li, 611731,China \\ ${ }^{2}$ The Joint Research Center for Satellite Navigation Sub-centers of The Ministry of Education, \\ University of Electronic Science and Technology of China, Yubai Li, 611731,China \\ ${ }^{3}$ The Joint Research Center for Satellite Navigation Sub-centers of The Ministry of Education, \\ University of Electronic Science and Technology of China, Yubai Li, 611731,China \\ ${ }^{4}$ The Joint Research Center for Satellite Navigation Sub-centers of The Ministry of Education, \\ University of Electronic Science and Technology of China, Yubai Li, 611731,China \\ aemail:photon_lee@uestc.edu.cn,bemail:741403318@qq.com, \\ cemail:309468723@qq.com, ${ }^{\mathrm{d}}$ email:ybli@uestc.edu.cn
}

Keywords: ADS-B, space borne, baseband processor, correlation.

\begin{abstract}
To resolve air traffic congestion and ensure flight safety, ADS-B provides a strong guarantee. As a collaborative associated monitoring system and an airborne aircraft navigation system, the main purpose of ADS-B is to periodically broadcast the aircraft-related parameters, such as call ICAO, longitude, latitude, altitude, speed, etc. However, the current terrestrial receiver baseband algorithm is the receiver baseband processing algorithms in ADS-B (1090ES) which is recommended in DO-260B aviation standards. Therefore, in this paper, I will introduce a new receiver baseband algorithm in space borne ADS-B (1090ES).
\end{abstract}

\section{Introduction}

The ADS-B technology is used to broadcast and access the latest flight data, and the apparatus are composed of a series of standard protocols. Based on the radio properties of the ADS-B, as long as within the range of the received signal, you can use the ADS-B ground receiving equipment, to receive the flight data and then to decode the data.

The biggest advantage of space borne ADS-B system is that it covers a wide range; however, there are some technical difficulties in this advantage:

(1)The airborne transmitter system does not make the appropriate improvements to adapt the space borne system, leading to that the signal power satellite received is very small. Designing a signal solver algorithm which is suitable for space borne environment is one of the technical challenges we are facing.

(2)Space borne ADS-B system is a single antenna coverage system. The coverage area is very large, in which there are a large numbers of aircrafts, and a large numbers of packets cause signal overlap situation seriously. How to separate the overlapping signals is another technical challenge we are facing.

The receiver baseband processing algorithms in ADS-B (1090ES) which is recommended in DO-260B aviation standards [1] cannot meet the requirements of the high sensitivity under the space borne environment for two reasons:

(1) We cannot set an effective threshold to make pulse have continuous four sampling points above this threshold.

(2) Waveform is smooth, transition amplitude is not enough, and it is difficult to find rising and falling edges.

In order to ensure the accuracy of the ADS-B data and to ensure the safety of aircraft flight, we need to improve the existing baseband algorithms. Here I come to introduce this new algorithm. 


\section{Receiver baseband algorithm in space borne ADS-B (1090ES)}

\section{A. Maximum likelihood criterion}

The key extraction of ADS-B signal is to identify effective pulse detection and its location. We mostly use maximum a posteriori estimation as a reference criterion of judgment.

In the binary detection, we suppose two different situations. Suppose $B_{0}$ corresponding to the pulse does not appear, and $B_{1}$ corresponding to the pulse appears. Assume $x$ as observations, we want to get each hypothetical probability values $P\left(B_{i} \mid x\right)$, i=0,1.If $P\left(B_{0} \mid x\right)>P\left(B_{1} \mid x\right)$, it is determined that the pulse is not detected; if $P\left(B_{0} \mid x\right)<P\left(B_{1} \mid x\right)$, it is determined that the pulse is detected. Assume $P\left(B_{0}\right)=\pi_{0}, P\left(B_{1}\right)=\pi_{1}$, with Bayes Theorem we finally get:

$$
\frac{p\left(x \mid B_{1}\right)}{p\left(x \mid B_{0}\right)}<\frac{D_{1}}{D_{0}} \frac{\pi_{0}}{\pi_{1}}
$$

Likelihood ratio is defined as:

$$
L(x)=\frac{p\left(x \mid B_{1}\right)}{p\left(x \mid B_{0}\right)}
$$

Because we do not know the priori probability of pulses, we define $\tau=\pi_{0} / \pi_{1}$. When $\tau=1$, it is called maximum likelihood criterion with threshold equals 1 . Assume the observed signal under $B_{i}$ is:

$$
\vec{x}=\vec{v}_{i}+\vec{y}
$$

$\vec{v}_{i}$ is each hypothesis corresponding complex set of values. $\vec{y}$ is collection of $k$ vice-zero mean Gaussian variables, all of whose features can be represented by the joint probability density, and the formula is as follows:

$$
p(\vec{y})=\frac{1}{(2 \pi)^{k} \operatorname{det}(M)} \exp \left(-\frac{1}{2} \vec{y}^{T} M^{-1} \vec{y}^{*}\right)
$$

Where $M$ is the plural covariance matrices $k$ order, $M$ is defined as:

$$
M=\frac{1}{2} E\left\{\vec{y}^{*} \vec{y}^{T}\right\}
$$

After simplification, we calculate the logarithm of $\mathrm{x}$, and finally we simplificate the formula and get:

$$
\operatorname{Re}\left\{\vec{x}^{T} \vec{h}^{*}\right\}_{<D_{0}}^{>D_{1}} \tau=\tau_{0}+\frac{1}{2}{\overrightarrow{v_{1}}}^{T} M^{-1}-\frac{1}{2}{\overrightarrow{v_{0}}}^{T} M^{-1}{\overrightarrow{v_{0}}}^{*}
$$

Among them, $\tau$ is a universal threshold, and:

$$
\vec{h}^{*}=M^{-1}\left(\vec{v}_{1}^{*}-\vec{v}_{0}^{*}\right)
$$

For ADS-B header pulse:

$$
\overrightarrow{v_{0}}=0
$$

So the universal optimal detector diagram is shown below:

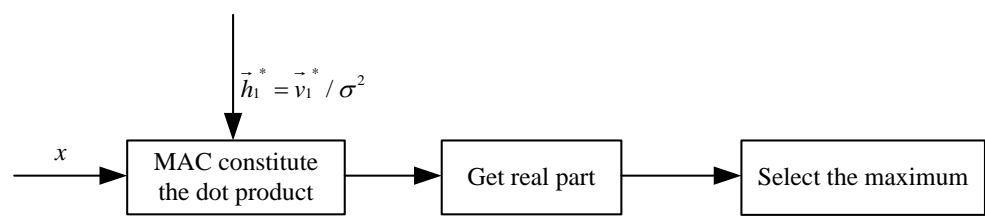

Fig.1 The universal optimal detector diagram

The general idea of the pulse detection is determined: The entering baseband signal is associated with the local pulse sliding window, and the largest peak found from the correlation results is the effective pulse VPP [2] desired position. 


\section{B. The process of the baseband receiver module in space borne ADS-B system}

Based on the maximum likelihood criterion in part A, we can find an effective pulse position VPP, which provides an important basis for finding the final effective pulse position FVPP in the next step. The improved process of the baseband receiver module is shown below:

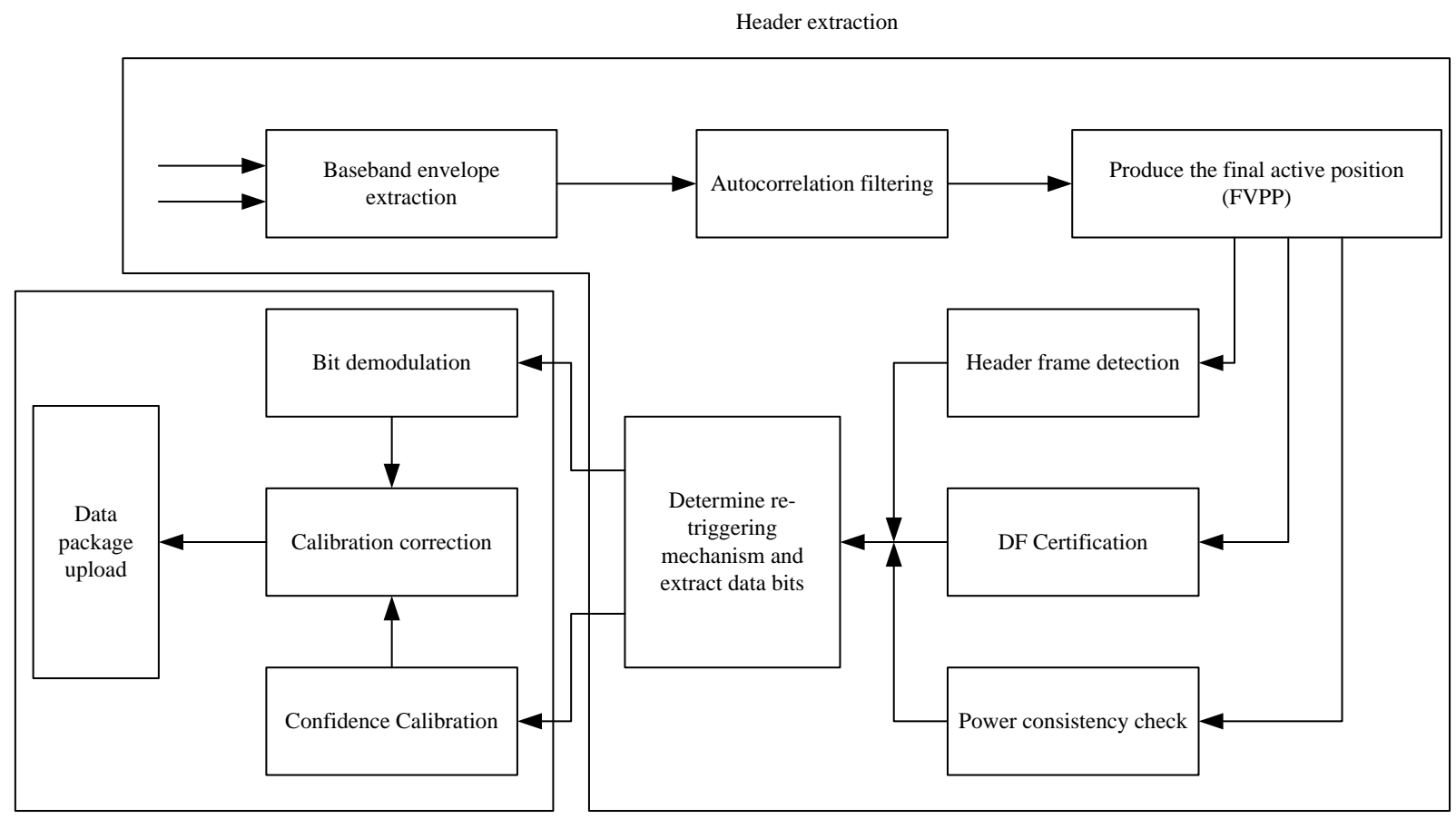

Data bits demodulation

Fig.2 The process of the baseband receiver module

It is divided into header extraction and data bits demodulation. We generate pulses, DF certification, frame detection, and power consistency criterion in the effective way. The new process is required to deal with the baseband signal and only extract FVPP, whose subsequent determination is based on a series of values and associated VPP without using VPP, REP and FEP.

\section{Baseband envelope extraction based on two quadrature signals}

In this paper, ADC (Analog Digital Convertor) gives the two orthogonal baseband signal, and we need to do further process if we want to get the baseband envelope.

The modulated ADS-B signal can be expressed as:

$$
x(t) \cos (2 \pi f c t+\varphi)=x(t) \cos \varphi \cos 2 \pi f c t-x(t) \sin \varphi \sin 2 \pi f c t
$$

After mixing with the local oscillation signal $1090 \mathrm{MHz}$ and low-pass filtering, we get two quadrature signals called I and Q, each of them is represented by:

$$
\begin{aligned}
& I(t)=x(t) \cos \varphi \\
& Q(t)=x(t) \sin \varphi
\end{aligned}
$$

So if we want to get the baseband signal envelope we need to find the square root of the two signals I and Q:

$$
x(t)=\sqrt{I(t)^{2}+Q(t)^{2}}
$$

$x(t)$ is the baseband envelope [3-4] we want to seek.

\section{Extraction algorithm based on the relevant valid pulse positions}

After introducing maximum likelihood guidelines, the original way to get APP, REP and FEP by analyzing the baseband signal has changed to the way to get FVPP by looking for the correlation peak position. The extraction procedure of FVPP is as follows: 


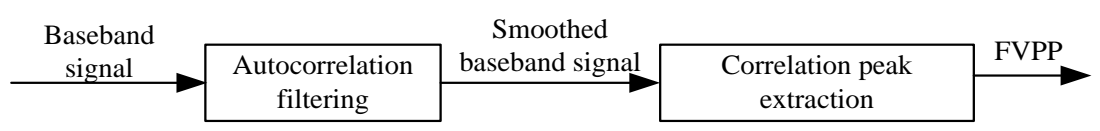

Fig.3 The extraction procedure of FVPP

The following is a detailed discussion of the above two steps:

(1) Autocorrelation filtering

The autocorrelation function of a signal reflects the signal strength between different moments. If we use signal to do autocorrelation process and select the appropriate parameters, it will be able to inhibit the noise of the mixed signal, so as to achieve the purpose of improving SNR [5].

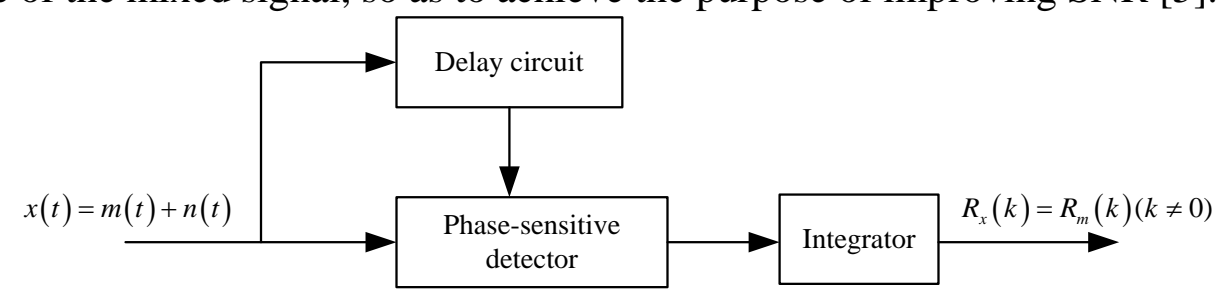

Fig.4 The framework of autocorrelation filtering

We additionally input signals as:

$$
x(t)=m(t)+n(t)
$$

Among them $m(t)$ is ADS-B baseband signal, $n(t)$ is additive white Gaussian noise signal whose mean is 0 . The autocorrelation function of $x(t)$ is:

$$
R_{x}(k)=E\{x(t) x(t+k)\}
$$

With the two functions above, we can get:

$$
R_{x}(k)=R_{m}(k)+R_{n}(k)+2 M_{m} M_{n}
$$

Since the autocorrelation function of white Gaussian noise is 0 when $k$ is not 0 , and it is 0 mean, we can get:

$$
R_{x}(k)=R_{m}(k)(k \neq 0)
$$

From the above equation we can see that the influence Gaussian white noise generates is eliminated in autocorrelation filtering, so as to achieve the purpose of smooth waveform [4].

(2)The extraction of FVPP with sliding window operation

After the baseband signal is filtered by autocorrelation, the waveform will be structured. The structured signal is related and match with the local pulse, determining the effective pulse position by looking for the correlation peak.

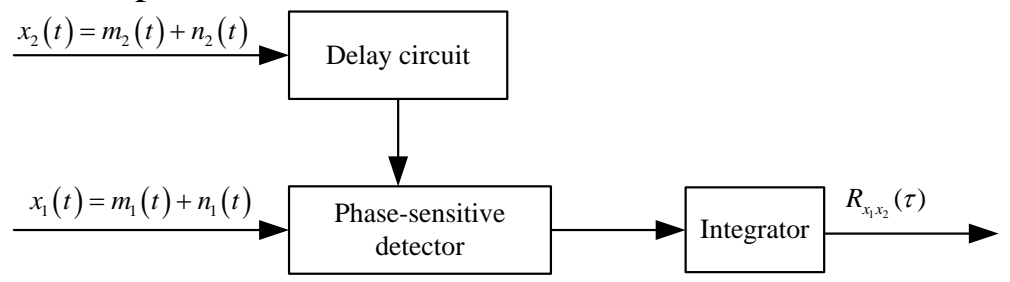

Fig. 5 The block diagram of cross-correlation process

Assume that the signal which needs to be cross-correlated is $x_{1}(k)$, the local relevant sequence is $x_{2}(k)$, and after the cross-correlation process the expression is:

$$
\begin{gathered}
R_{x_{1} x_{2}}(\tau)=E\left(x_{1}(t) x_{2}(t-\tau)\right)=\lim _{T \rightarrow \infty} \frac{1}{T} \int_{-T / 2}^{T / 2} x_{1}(t) x_{2}(t-\tau) d t \\
=R_{x_{1} x_{2}}(\tau)+R_{x_{2} n_{2}}(\tau)
\end{gathered}
$$

As the signal and noise are not correlated, we can get $R_{x_{1} n_{1}}(\tau)=0$. Then we can extract the peak position to determine a final valid pulse position FVPP [6].

When we extract FVPP we must avoid the interference of the false correlation peak. We use the following guidelines to extract the effective pulse position(assume that $X_{-3}, X_{-2}, X_{-1}, x_{0}, x_{1}, X_{2}, X_{3}$ is 
the 7 sampling points in the sliding window).

1. $x_{0} \geq x_{1} \geq x_{2} \geq x_{3}$ and $x_{0} \geq x_{-1} \geq x_{-2} \geq x_{-3}$;

2. $x_{0} / x_{3} \geq 1.5$ or $x_{0} / x_{-3} \geq 1.5$;

3. $x_{0} \geq T h$;

4. While meeting the above 1,2 , and 3 points $x_{0}$ can determine a effective pulse position.

\section{E. The improvement of frame detection mechanism}

Based on the ADS-B message transmission waveform, in message header there are four pulses on $0,1.0,3.5$, and $4.5 \mu \mathrm{s}$. So we can use the timing relationship of the header pulse to detect the header. We use the frame detection mechanism based on FVPP instead of the frame detection mechanism based on VPP-REP-FEP. The detection condition of FVPP is more strict than VPP, so we relax the determination standard of frame detection allowing that 2 sampling points can appear hop in one of the positions.

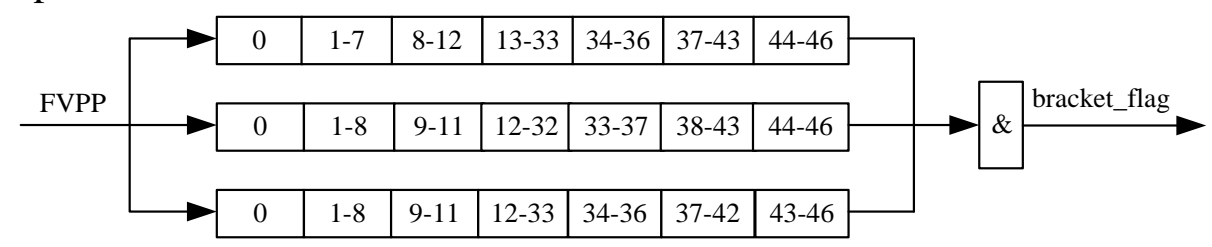

Fig.6 The schematic diagram of improved frame detection mechanism

\section{F. The improvement of DF determination}

The authentication mechanism of DF is putting the demodulation of some data bits (the first 5 bits) into the header detection. We directly demodulate the DF bit to get the first 5 data bits. For 1090ES data link we are concerned only with the packets of DF $=17$ (the binary is 10001). ADS-B (1090ES) packet baseband modulation scheme is PPM modulation: if the pulse in the first half symbol period represents bit 1 , the other represents bit 0 . So we take the sample points from $8 \mu$ s to $15 \mu$ s to do pre-demodulation. As an example of the sampling rate of $10 \mathrm{M}$ one symbol period has 10 sampling points, and we set them as $x_{0}, x_{1}, x_{2}, x_{3}, x_{4}, x_{5}, x_{6}, x_{7}, x_{8}, x_{9}$. When $\sum_{i=0}^{4} x_{i} \geq \sum_{i=5}^{9} x_{i}$, the bit is determined as 1 ; when $\sum_{i=0}^{4} x_{i}<\sum_{i=5}^{9} x_{i}$, the bit is determined as 0 . If the demodulation results is $\mathrm{DF}=$ 17, we pass the determination and enter the re-trigger process.

\section{G. The improvement of power consistency judgment}

Compared to the original algorithm, the main difference is in the relevant average value calculation and the reference power value calculation which each pulse is correspond to. In the improved algorithm, we directly extract the header value of the sliding window correlation signal which 4 FVPP correspond to and calculate the average value as the reference power. The value is the reference power value of each pulse.

Consistency power detection algorithm is described as follows:

(1) Extract the cross-correlation value of the four pulse position corresponding to the signal $x_{p_{1}}, x_{p_{2}}, x_{p_{3}}, x_{p_{4}}$;

(2) Calculate the average value $x_{\text {averge }}$;

(3)If $x_{p_{1}}, x_{p_{2}}, x_{p_{3}}, x_{p_{4}}$ is in the value of $x_{\text {averge }}$ which pluses or minuses $3 \mathrm{~dB}$, it is determinated effectively. Otherwith, it is not determinated effectively. 


\section{Simulation and verification}

\section{A. The comparison of the filtering effect using autocorrelation filter under different orders}

For ADS-B signal, the width of header pulse is $0.5 \mu \mathrm{s}$, and it is five sampling points in $10 \mathrm{MHz}$ sampling rate. The following are the simulation using a sampling rate of $10 \mathrm{MHz}$.
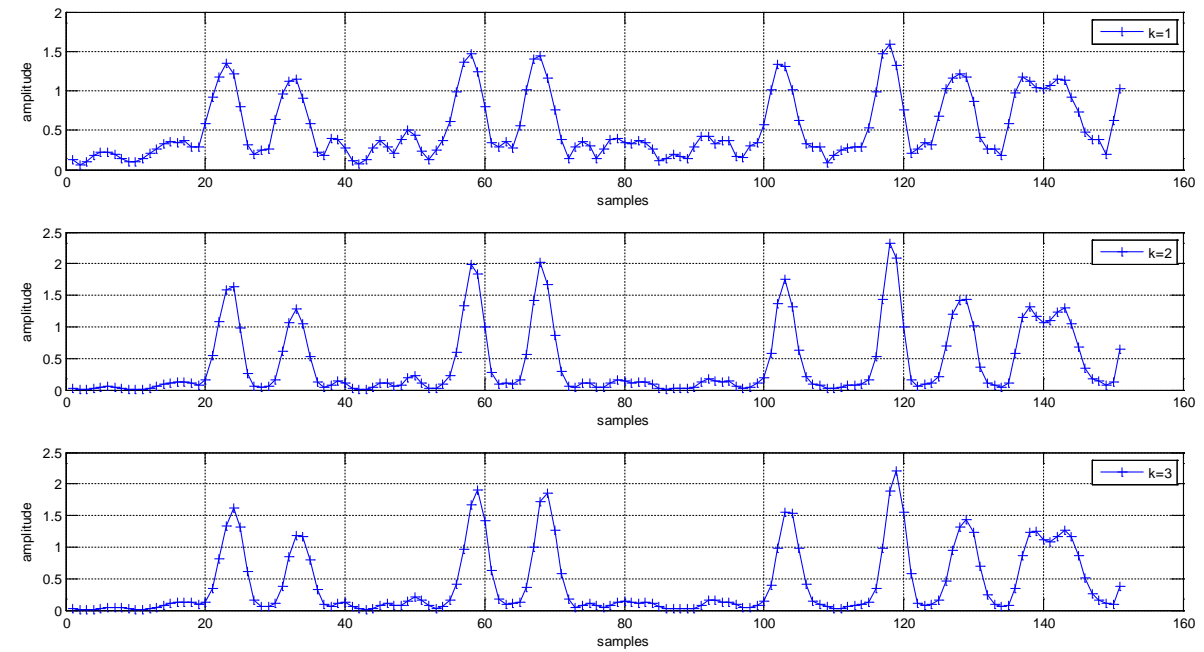

Fig.7 The comparison of the filtering effect using autocorrelation filter under different orders

We found that when $\mathrm{k}=3$ the waveform is the steepest, the distinction of the header pulses is the most obvious, and 3 order is only bigger than 2 order. In comprehensive comparison we set autocorrelation order to 3 .

\section{B. The extraction of FVPP using sliding window}

For the four preamble pulses of ADS-B signal, the pulse width is $\Delta \tau=0.5 \mu \mathrm{s}$. The baseband signal will first enter a sliding window whose depth is 5 . At each time we slide only once, the data in the window will get a correlation value related with the local signal sequence.

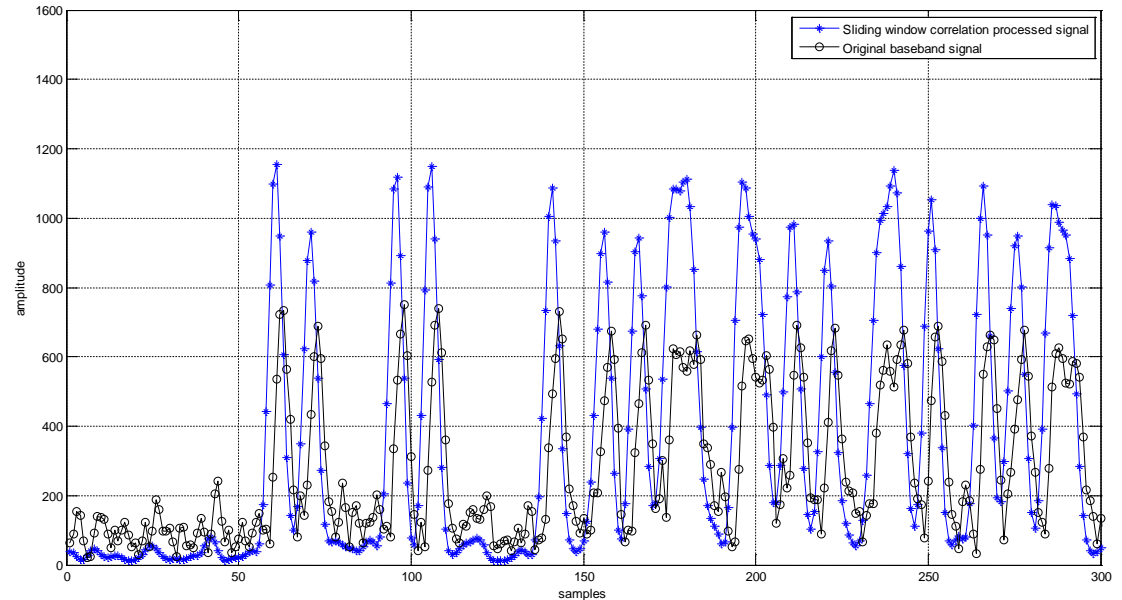

Fig.8 The comparison of baseband waveform before and after correlation

The blue signal is a baseband envelope signal after autocorrelation filtering process, and the black signal is a waveform after sliding window correlation. With comparison, we can found that after correlation process the waveform is further smooth, and the number of extreme points further reduces.

\section{The analysis and comparison of header detection algorithm}

Compared to the original algorithm the extraction of FVPP also needs to preprocess the baseband 
signal. Under the condition that the SNR is high and the signal quality is good, the effect is not obvious whether we do autocorrelation filtering or not. However, when the SNR deteriorates to 3dB the situation becomes different, and the specific situation is as shown in figure 9.
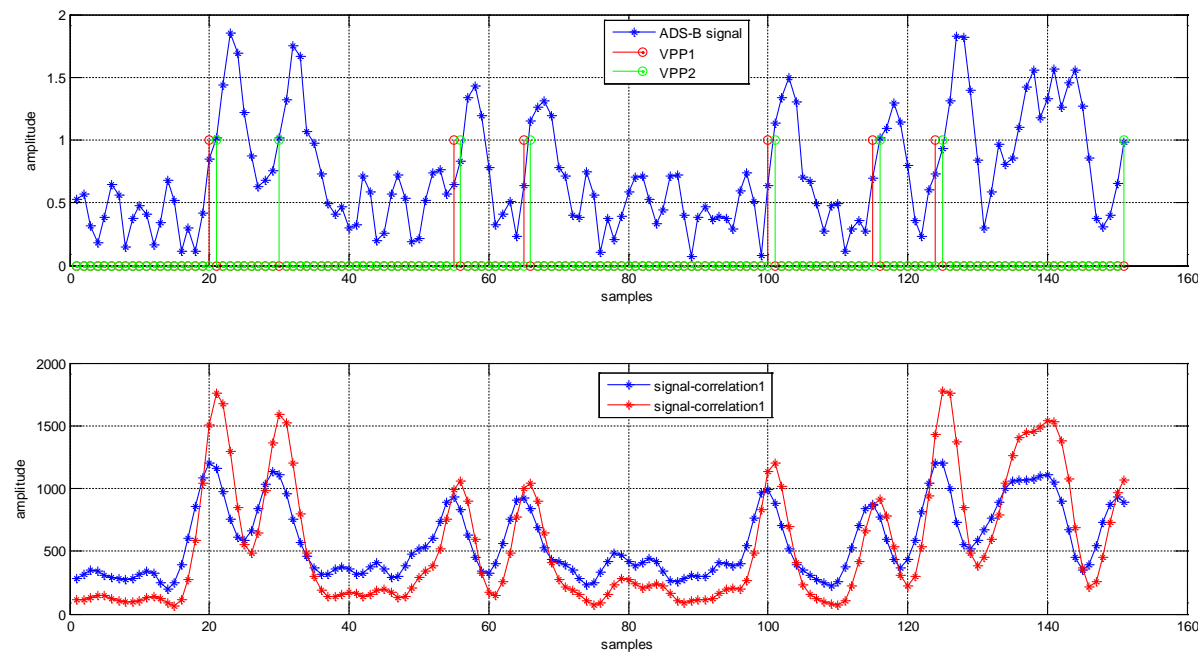

Fig.9 The comparison of cross-correlation results before and after autocorrelation filtering

The red waveform is processed by autocorrelation filtering and then sliding window, and the blue waveform is only processed by sliding window. Clearly, the correlation peak of the red waveform is more obvious than the blue waveform's, so the success rate of the extraction of FVPP is higher. The root cause of this situation is the inhibition of noise with autocorrelation filtering.

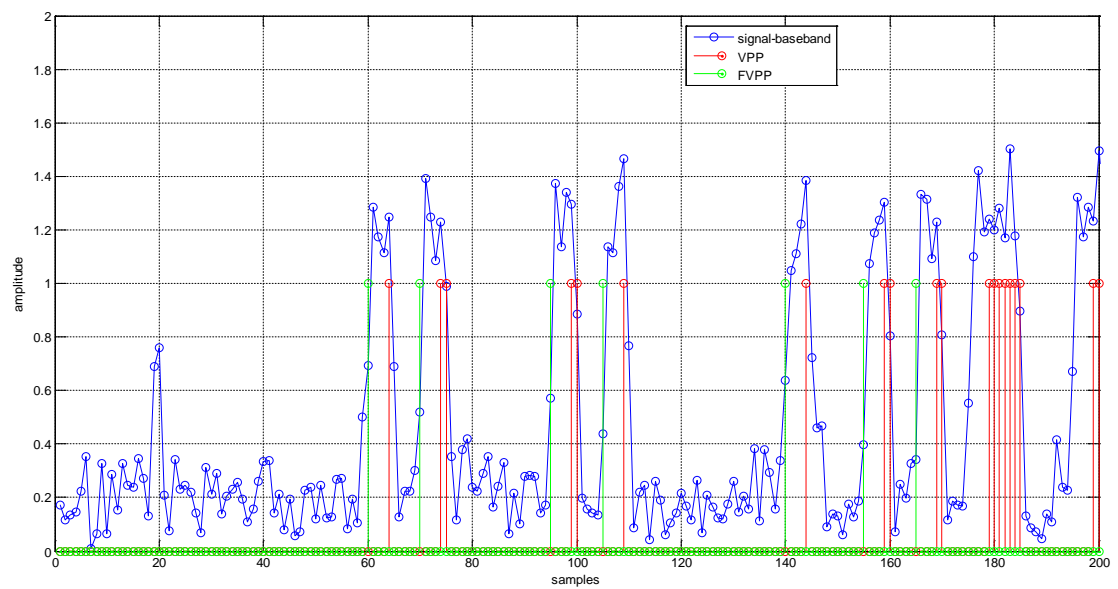

Fig.10 The comparison of the extraction of VPP and FVPP (SNR=9dB)

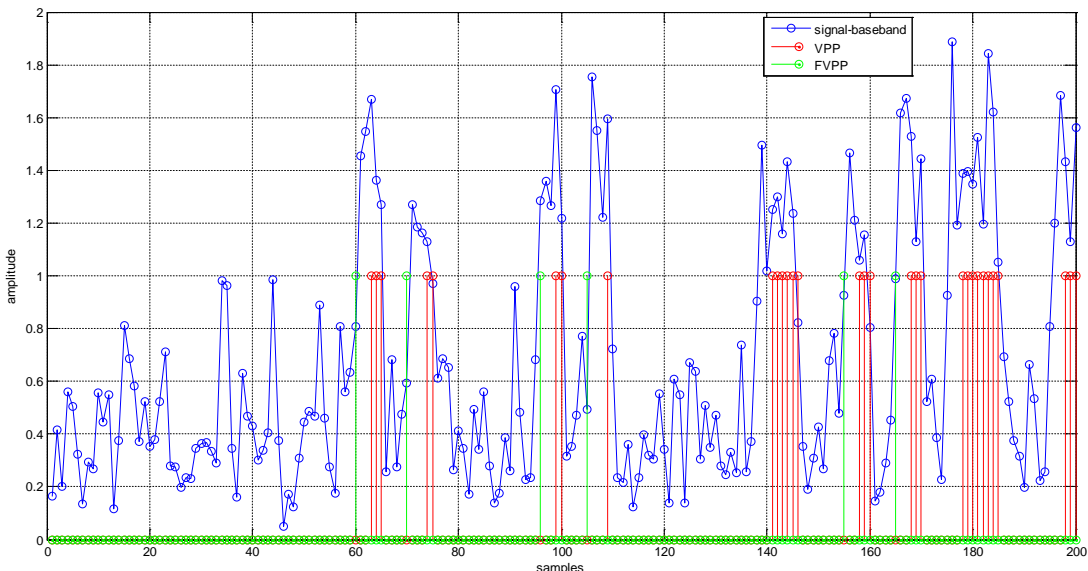

Fig.11 The comparison of the extraction of VPP and FVPP (SNR=3dB) 
When the SNR further deteriorates, FVPP is still able to be correspond with the header pulse at 3dB SNR. The reason is that the baseband signal bandwidth becomes narrow which leads to waveform distortion, and the way in which the threshold is originally set to determine VPP becomes difficult to work.

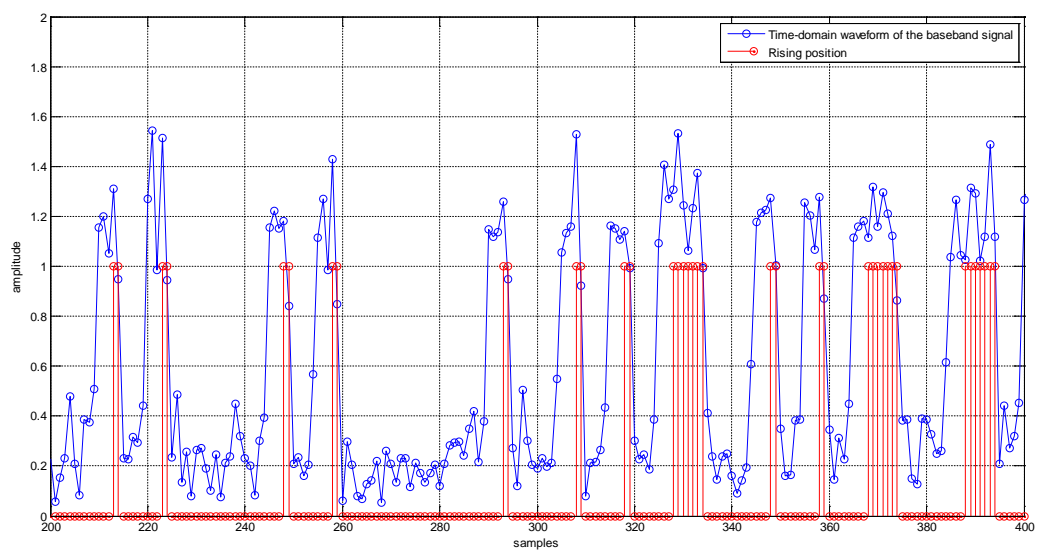

Fig.12 The extraction of rising edge (SNR=9dB)

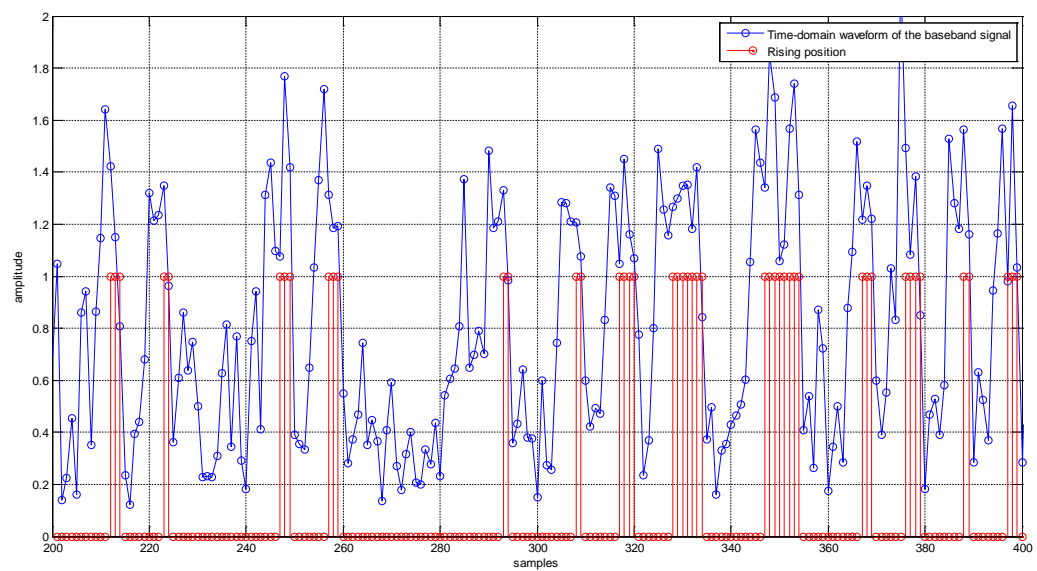

Fig.13 The extraction of rising edge (SNR=3dB)

When SNR is $9 \mathrm{~dB}$, the REP of 4 pulses can be extracted, but it would extract much invalid rising edge at the same time. When SNR is $3 \mathrm{~dB}$, only at 1 position we can extract the rising edge.

\section{Header extraction performance comparison of the improved algorithm and the original algorithm}

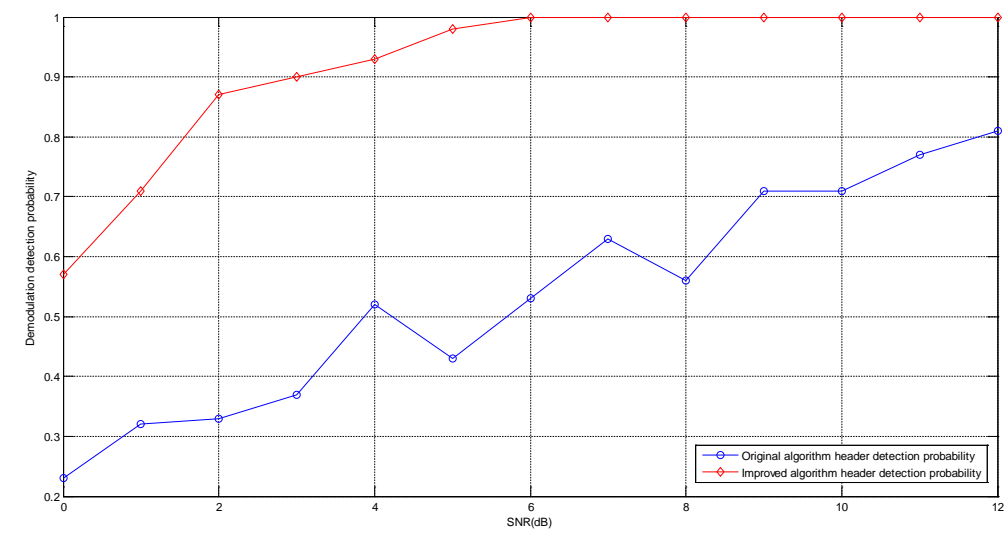

Fig.14 Detection performance comparison before and after the method improved

We can see that when the SNR is $6 \mathrm{~dB}$ the header detection rate of the improved algorithm is more than $95 \%$, while the performance of the original algorithm is very poor in adapting to the 
narrow band and its header detection rate is less than $90 \%$ when SNR is in the range of 0dB and $12 \mathrm{~dB}[7-9]$.

\section{Conclusion}

In the continuation of the first frame detection in DO-260B aviation standards, we then do DF certification, verification of the consistency of power, re-triggering etc. The great improvement in space borne algorithm is that it introduces maximum likelihood coherent detection into the extraction of FVPP. In the process, we use correlation to extract FVPP. The following is a specific aspect of improvement: the extraction of effective pulse position; the improvement of frame detection mechanism; the improvement of DF determination; the improvement of power consistency judgment.

\section{Reference}

[1] RTCA. DO-260B. Minimum Aviation System Performance Standards for Automatic Dependent Surveillance Broadcast(ADS-B)[S]. 2006

[2] Li Xiaofeng. The Communication Theory [M]. Beijing: Tsinghua University Press, 2008, $199-213$

[3] Cao Zhigang. The Modern Communication Theory [M]. Beijing: Tsinghua University Press, 1992, 152-162

[4] Guo Tao. The Key Technology Research Of ADS-B System Based On Transmission And Reception Which Are In An Entirety In Mode S [D]. Chengdu: University Of Electronic Science And Technology, 2013

[5] Li Rui, He Fuyun, Xia Yubao. The Principles And Applications Of Correlation Detection [J]. The Learned Journal Of Hefei University Of Technology, 2008, 31(4): 573-575

[6] Xu Xiao, Li Yuan. MATLAB Object-oriented Programming - From Entry To Design Mode [M]. Beijing: Beijing University Of Aeronautics And Astronautics Press, 2014, 67-98

[7] Zhang Zhiyong. MATLAB Tutorial R2010a [M]. Beijing: Beijing University Of Aeronautics And Astronautics Press, 2011, 33-54

[8] Xu Xiao, Li Yuan. MATLAB Object-oriented Programming - From Entry To Design Mode [M]. Beijing: Beijing University Of Aeronautics And Astronautics Press, 2014, 56-78

[9] Huang Hai. The Processing Board Design Of LFM Radar Signal Based On TMS320C6416 [D]. Chengdu: University Of Electronic Science And Technology, 2005, 30-31 